\title{
Retinopathy of prematurity: oxygen dogma challenged
}

\author{
'The report of my death was an exaggeration': Mark Twain in a cable to \\ Associated Press
}

The euphoria after the demonstration in autumn $1954^{1}$ of a firm link between prolonged exposure to high concentrations of oxygen and an increased risk of retrolental fibroplasia lasted nearly a quarter of a century. Before 1954 there had been intense debates concerning the role of supplemental oxygen. The calm that followed was remarkable: hardened sceptics raised few objections to the subsequent claims and demands for practical action even though these new assertions concerning oxygen restriction went well beyond the narrow limits of evidence which had undergone rigorous testing. ${ }^{2}$ For example, in 1955 a memorandum sent to all health officers in the state of New York declared, 'blindness due to retrolental fibroplasia appears to be entirely preventable ... if premature infants are not exposed to high concentrations of oxygen' ${ }^{3}$ Paediatricians were told 'the concentration of oxygen should never exceed $40 \%,{ }^{4}$ and later, ' . . retrolental fibroplasia can be either completely or almost completely eliminated by administering oxygen only at times of clinical need, and then for as brief periods as possible and at concentrations less than $40 \%{ }^{5}$ These words, with little modification, soon found their way into bulletins, health codes, and official recommendations, and into textbooks of paediatrics and ophthalmology.

The precipitous fall in occurrence of retinopathy of prematurity (ROP) (the term preferred in recent years) throughout the world after the adoption of recommendations for oxygen restriction was taken as convincing evidence that hyperoxia was, indeed, the sole necessary and sufficient cause of the retinal disorder. Opinion as to cause and effect was soon so rigid that every example of ROP blindness was taken as proof of improper use of supplemental oxygen. Needless to say, this incriminatory attitude served to immunise the oxygen thesis against criticism (and it proved to be a bonanza for tort lawyers). The inverted reasoning reached what must be considered the very apogee of credulousness when the eye complication in a $1140-\mathrm{g}$ twin was blamed on the administration of oxygen for 275 minutes during surgery at age 3 days. $^{6}$ The editorial accompanying the anecdote warned: 'the experience to date suggests that the most susceptible infants can be blinded by a total exposure of just 2 or 3 hours to a $\mathrm{PaO}_{2}$ greater than 100 torr . . . anesthetists . . . must realise they can blind such an infant with excess oxygen during the few hours the infant is in their care.'?

Quite apart from the fact that 'the experience' quoted consisted of lonely numerators looking for unavailable denominators, the peremptory comments ignored challenging questions which had been accumulating during years of dogmatic slumber. For example-why does eye damage occur in such a small minority of preterm infants who experience prolonged hyperoxia?; why does the disorder occur in some babies (including stillborns) who have never been treated with supplemental oxygen?; why has it been so difficult to establish a correlation between $\mathrm{PaO}_{2}$ levels and blindness risk?; why has it been impossible to blind experimental animals by exposure to supplemental oxygen alone?; and, most disturbing of all, why do the numbers of affected children seem to be rising at a time when there has been pronounced improvement in the technical methods of oxygen delivery and monitoring ? ?-10 $^{-10}$ It is, of course, this last question which is a forceful reminder that (1) ROP was never totally eliminated by the policy of oxygen restriction, (2) epidemiological evidence suggested, in retrospect, that the very low incidence of ROP blindness during the first decade of oxygen restriction was related to the fact that neonates at greatest risk of developing the condition (the smallest infants, ${ }^{11}$ especially those with respiratory distress syndrome ${ }^{12}$ ) were dying in the first hours of life, the restrictive policy's much touted accomplishment may have been a Pyrrhic victory, and (3) factors operating to determine the infrequent switch from reversible vascular changes in the eyes of preterm infants to an unremitting cicatricial retinopathy have never been understood.

The watertight case in support of 'hyperoxia is the cause of ROP' has been leaking for years, the leaks are now so large they can no longer be dismissed. It is encouraging to see that the unsatisfactory state of affairs has stimulated a lively interest in the subject and renewed investigations. Among these are revived studies of a possible protective role of the antioxidant, vitamin $\mathrm{E}$; results in the newborn kitten lend some indirect support for the weakened 
oxygen theory. For example, Phelps and Rosenbaum's experiments have suggested that abnormal vascular changes in the retina induced by oxygen exposure are ameliorated by concurrent treatment with tocopherol. ${ }^{13}$ And Bougle et al..$^{14}$ showed that in the retina of kittens hyperoxia leads to a fall in an important enzyme (superoxide dismutase) concerned with protection against the toxic effects of oxygen radicals; the drop in superoxide dismutase appears to be partially prevented by pretreatment with a large dose of vitamin $\mathrm{E}$. The experimental results in kittens are provocative, but, unfortunately, it is hard to know whether they have anything to do with the problem of blindness in infants; as already noted cicatricial lesions cannot be induced in animals by exposure to oxygen alone. Moreover, the beneficial effect of vitamin $E$ in the kitten model of retinopathy may not be entirely specific (that is to protect against oxygen toxicity). Phelps and Rosenbaum have now shown suppression of oxygen-induced vitreal neovascularisation when tocopherol was first given after the animals were removed from oxygen..$^{15}$

Unfortunately, the riddles have not been resolved by clinical studies of vitamin $E$ treatment of preterm infants. The results of several small-scale randomised control trials have suggested that prophylactic administration of tocopherol has an effect in reducing the severity of outcome in ROP. However, the conclusions are far from convincing because of problems related to design and size of sample ${ }^{16}$ in reported trials, and there are unresolved questions about the safety of administering pharmacological doses of tocopherol to large numbers of preterm infants. ${ }^{17}$

Two recent sets of animal experiments have added fresh fuel to the fires of controversy which now surround the oxygen dogma. In the first of these the conventional wisdom concerning strict oxygen restriction was challenged by Phelps and Rosenbaum. ${ }^{18}$ They noted that infants who develop severe eye damage have lower $\mathrm{PaO}_{2}$ levels than others without cicatricial ROP and proposed that a restrictive policy might be responsible for precipitating the serious form of the disorder. The hypothesis seemed to be supported by an experiment on two groups of kittens exposed to a standard oxygen exposure $\left(\mathrm{F}_{\mathrm{IO}} \mathrm{O}_{20} \mathrm{\%}\right.$ for 65 hours): the animals who spent the next 28 days in hypoxic conditions had more severe retinal changes than controls who were placed in room air for a month after hyperoxic exposure. The authors warned that (1) extrapolation from the animal model was limited by the fact that no cicatricial lesions were produced, and (2) these experimental results were not ready to be tested in human trials (and should not be translated into clinical action!) until further explorations of the hyperoxia-hypoxia phenomenon in the kitten were completed.

The second set of animal experiments has given results which, if confirmed, threaten to overturn many accepted views. Flower and associates examined the effect of blocking oxygen-induced vasoconstriction with aspirin (to alter prostaglandin biosynthesis) and were able to produce cicatricial retinopathy.$^{19}$ For the first time, the authors noted, a scarring lesion was produced in an experimental animal and this seemed to have been accomplished by interfering with a change, retinal vasoconstriction, heretofore considered to be the first stage of a sequence leading to retinal disease. Similar effects were produced when hypercarbia was maintained during chronic oxygen exposure and this has led to the speculation that retinal vasoconstriction is a normal physiological mechanism to protect the immature retina from the damaging effects of hyperoxia. According to this thesis retinal damage occurs only in infants with inadequate retinal vasotonia for protection of structurally immature vessels. Most importantly, the creation of cicatricial lesions in an animal eye answers the major criticism of prehuman studies and, if reproduced by others, the feat will strengthen the confidence with which the results are extrapolated to ROP blindness in infants. These landmark observations also suggest a mechanism (increased blood flow and raised transluminal pressure in the developing retinal vasculature) to explain how scarring complications can occur without exposure to supplemental oxygen.

The present uncertainties may come as a shock to those who were taught that the story of ROP blindness is a closed, and satisfactorily concluded, chapter in the early history of modern neonatal medicine. But the latest turn of events is not unique nor is the current state of disbelief in the oxygen dogma particularly worrying. (The maxim, 'Better orderly error than complex truth' is dangerous to our patients' health.) In fact, the general problem faced by physicians of proceeding from 'the scanty field of what is known ... . into the boundless region of what is unknown' was noted 175 years ago by the polymath Thomas Jefferson, third president of the United States. ${ }^{20} \mathrm{He}$ cautioned the 'adventurous' doctors of his day to recall that 'the natural course of the human mind is certainly from credulity to scepticism'.

\section{References}

1 Kinsey V E. Retrolental fibroplasia. Cooperative study of retrolental fibroplasia and the use of oxygen. Arch Ophthalmol 1956; 56: 481-543. (Note: the results of this study were made public in September 1954.) 
2 Anonymous. Oxygen and retrolental fibroplasia: the questions persist. Pediatrics 1977; 60: 753-4.

3 Yankauer A. Information memorandum from New York State of Health. 17 February 1955.

4 Lanman J T. The control of oxygen therapy for premature infants. Health News 1955; 32: 14-6.

5 Guy L P, Lanman J T, Dancis J. The possibility of total elimination of retrolental fibroplasia by oxygen restriction. Pediatrics 1956; 17: 247-9.

- Betts E K, Downes J J, Schaffer D B, Johns R. Retrolental fibroplasia and oxygen administration during general anesthesia. Anesthesiology 1977; 47: 518-20. (Note: the eyes of the affected child's co-twin remained normal despite exposure to high $\mathrm{FJO}_{2}$ for 79 hours; radial artery $\mathrm{PaO}_{2}$ in both infants ranged widelyperiods ( $\mathrm{f}$ hyperoxemia, over 150 torr, were documented in both twins.)

7 Phibbs R H. Oxygen therapy: a continuing hazard to the premature infant. Anesthesiology $1977 ; 47$ : 486-7.

8 Gunn T R, Aranda J V, Little J. Letter: Incidence of retrolental fibroplasia. Lancet 1978 ; i: 216-7.

9 Tarkkanen A, Mustonen R. Retrolental fibroplasia: cases seen at the Helsinki University Eye Hospital 1956-74. Acta Ophthalmol (Copenh) 1978; 56: 169-78.

10 Phelps D L. Retinopathy of prematurity: an estimate of vision loss in the United States-1979. Pediatrics 1981; 67: 924-6.

11 Cross $\mathrm{K} \mathrm{W}$. Cost of preventing retrolental fibroplasia? Lancet 1973; ii: 954-6.

12 Avery M E, Oppenheimer E H. Recent increase in mortality from hyaline membrane disease. $J$ Pediatr 1960; 57: 553-9.
13 Phelps D L, Rosenbaum A L. The role of tocopherol in oxygen-induced retinopathy: kitten model. Pediatrics 1977; 59: 998-1005.

14 Bougle D, Vert P, Reichart E, Hartemann D, Heng E L. Retinal superoxide dismutase activity in newborn kittens exposed to normobaric hyperoxia: effect of vitamin $E$. Pediatr Res 1982; 16: 400-2.

15 Phelps D L, Rosenbaum A L. Vitamin E in kitten oxygeninduced retinopathy. Arch Ophthalmol 1979; 97: 1522-6.

16 Chalmers T C, Smith H, Blackburn B, et al. A method for assessing the quality of a randomized control trial. Controlled Clin Trials 1981; 2 : 31-49.

17 Kalina R C, Hillis A, Sepkowitz S, Sobel S, et al. and Hittner $H$ M. Letters: Vitamin $E$ and retrolental fibroplasia. $N$ Engl J Med 1982; 306: 866-8.

18 Phelps D L, Rosenbaum A L. The effect of marginal hypoxemia during the recovery period in oxygen-induced retinopathy in the kitten (abstract). Clin Res 1982; 30: 146A.

19 Flower R W, Blake D A. Retrolental fibroplasia: evidence for a role of the prostaglandin cascade in the pathogenesis of oxygen-induced retinopathy in the newborn beagle. Pediatr Res 1981; 15: 1293-302.

20 Jefferson T. Letter to Doctor Caspar Wistar, 21 June 1807. Cited in The works of Thomas Jefferson in twelve volumes, federal edition. New York: Putnam, 1904-5: 423.

William A Silverman 90 La Cuesta Drive, Greenbrae, California 94904,

USA 